\title{
Modern information and telecommunication systems for medical purposes
}

\author{
Oleksii Serheiev-Horchynskyi, ${ }^{1, *}$ \\ ${ }^{1}$ NTUU "Igor Sikorsky KPI", Inst. for Applied System Analysis, Dept. of Sys. Design, 37 Prosp. Peremohy, 03056, Kyiv, Ukraine
}

\begin{abstract}
The analysis and classification of modern information and telecommunication systems (ITS) for medical purposes were carried out. Historically established areas of application of medical telecommunication systems were distinguished. Classes of communication and data related to modern medical telecommunication systems were analysed. The role of standards and protocols for data transmission in the process of interaction between the hardware and software components of medical ITSs was defined. Standards for transmitting various types of information were listed; the exploration of the standards for telemedicine information transmission was carried out. Various structural-logical schemes of medical ITSs implementation were illustrated; components of the schemes were reviewed. The importance of development and implementation of a unified interaction model for various medical ITS components, that will allow adapting to extreme characteristics of data transmission physical environment, was figured out.
\end{abstract}

\section{Introduction}

The classes of medical ITSs and structural-logical schemes of their implementation vary due to a wide range of used medical data types $[1 ; 2]$, specifications of the implementation of data transmission protocols, parameters of the hardware implementation, and so on. The underlying issue of effective exploitation of ITSs from the beginning of their development up to the present time is the noise immunity of data transmission [1].

It is clear that in emergencies, specialized medical systems become strategically crucial in the struggle for human life. The general objective of remote medicine is to provide medical services based on ITSs of various classes, demanding that the quality of medical data exchange at all levels including registration, transmission, and storage of medical data is guaranteed $[1 ; 2]$.

For remote areas, the issue of transmission of telemedicine information about a person's condition over a distance is essential and involves the provision of storage and processing of the medical data $[1 ; 3]$. The use of remote access technologies in medical ITSs results in improved quality and speed of providing medical services, especially in areas of social disasters with a damaged medical infrastructure.

\section{Classification of Medical Data and Telecommunication Systems}

\subsection{Areas of Medical ITSs Application}

The following historically established main areas of application of information and telecommunication technologies in medicine can be distinguished [3-6]:

- telemedicine consultations;

- patient video surveillance;

- telemedicine of urgent conditions, and emergencies;

- tele-education;

- telesurgery and operative prevention;

- space telemedicine.

The most common application of medical ITSs is remote consultations, which can use both static (video, audio, text) and dynamic (conferencing) information.

\subsection{Medical Data}

Modern medical conferencing can be segmented into two classes: consultations (point-to-point mode) and councils (multipoint mode), providing for direct communication between the patient, doctor, and consultant in near realtime mode. In both cases, the transmission of medical data requires a stable process at high levels of interference.

Medical data cover a wide range of critical information needed to make informed decisions about a patient's health state. Assessments of the patient's condition are usually performed based on requests to the electronic database of the history of diseases, the results of recent clinical tests, visual data about the patient's condition (radiograms, computer tomograms, ultrasound images, and so on).

The static resources, which are analysed in off-line mode, don't require high-speed communication channel and specialized hardware [1]. Unlike static, providing a conference communication (based on dynamic resources) requires the installation of special equipment (digital video and audio equipment for data acquisition and

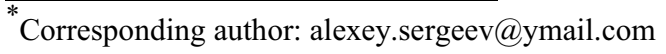


transmission) with access to broadband communication technologies, the use of specialized software and specialized data exchange protocols [3].

Medical ITSs (systems for dynamic monitoring) allow registering the physiological and biometric parameters of the patient's body and transmitting them to the base station for further analysis. Such systems are already used for patients who require frequent medical screening and continuous physiological monitoring $[1 ; 3]$.

For data transmission, current medical ITSs use $3 \mathrm{G}$, 4G, Wi-Fi, WiMAX, LTE standards. Satellite systems, including the International Maritime SatelliteCommunication System, are used to deliver critical data from remote areas [3].

\section{Standards}

\subsection{Principles of Information Transmission}

Essential telemedicine information can be classified as follows [6-8]: documents in electronic form; visual images; recordings of electrical signals; multimedia information; information in the video conferencing mode. The interaction and data exchange between components of ITS are governed by the standards and protocols.

The names of the standards include the abbreviations of the groups, organizations or institutions involved in their development [8]. For instance, ASC X12N deals with external standards for the exchange of electronic documents, ASTM E31.11 - exchange of laboratory test data, IEEE P1157 - exchange of medical data (MEDIX), ACR-NEMA DICOM - exchange of video/audio data.

Modern medical ITSs use various medical and communication hardware, specialized communication interfaces and protocols [9]. There is an urgent need to develop and implement a unified model of interaction of medical equipment, allowing adapting to any characteristics of the physical environment for data transmission.

\subsection{Standards for Information Transmission}

Below are essential and most commonly used standards for the transmission of information, taking account of their adaptation to medical ITS [10].

Standards for telemedicine data transmission developed by the International Organization for Standardization (ISO):

- ISO 5218 (1977) - exchange of data, representation of the gender of a person;

- ISO 1000 (1981) - units of measurement, SI system;

- ISO 2955 (1983) - processing of data for the representation of SI units and other units in systems with a limited set of characters;

- ISO 8072 (1986) - networking standards;

- ISO 8601 (1988) - data elements and exchange formats, date and time representation;

- ISO 8859 (1988) - data processing, a table of 8-bit single-byte character codes.
Standards developed by the American National Standards Institute (ANSI):

- ANSI X3.30 (1985) - representation of a calendar and ordinal date;

- ANSI X3.4 (1986) - a table of character codes, the American Standard Code for Information Interchange (7bit ASCII table);

- ANSI X3.43 (1986) - representation of the local time of day for data exchange in information systems;

- ANSI X3.50 (1986) - representation of SI units, traditional U.S. units and other units in systems with a limited set of characters;

- ANSI X3.51 (1986) - representation of universal time, local time shifts and U.S. time zones for data exchange.

Standards developed by the American Society for Testing and Materials (ASTM):

- ASTM E31.12 (1990) - representation of clinical tests;

- ASTM E1467 (1994) - transmitting digital neurophysiological data in computer systems;

- ASTM E1394 (1997) - exchanging data between diagnostic equipment and computer systems;

- ASTM E1381 (2002) - transmitting low-level data between clinical laboratory and computer systems.

The specific standards for the exchange of medical data are described below.

\subsection{HL7 Standard}

HL7 (Health Level 7) - approved in 1996 the United States' national standard for the exchange of medical data in electronic form $[11 ; 12]$, see Fig. 1.

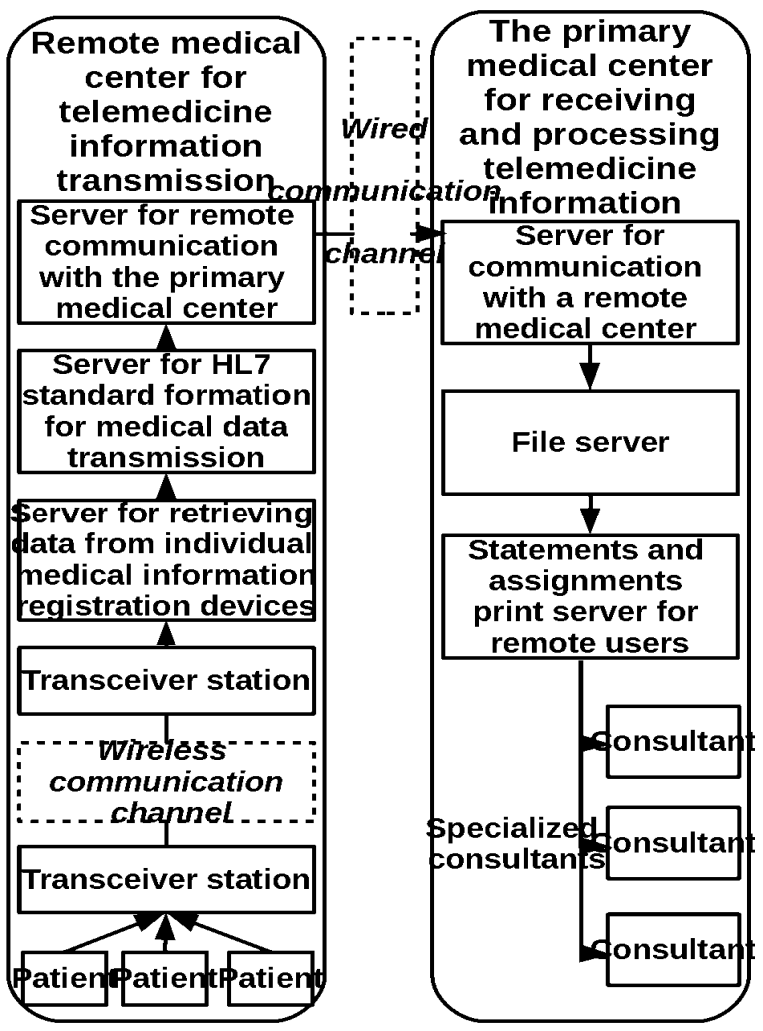

Fig. 1. Structural-logical scheme of organization of medical ITS using the HL7 standard. 
The objectives of the standard HL7 were: to provide the interoperability of automated systems in health care facilities; to unify the exchange of external data; to standardize data exchange between medical ITS (standardization of software interfaces); to support digital data exchange across a wide range of communication environments [12].

The term "Level 7" refers to the top level of the Open Systems Interconnection (OSI) model. The standard includes the definition and structure of data transmission, synchronization of medical data exchange, and standard principles of communication systems' provision based on the noise-resistant data encoding/decoding.

The HL7 standard defines the mechanism of addressing to the interfaces of various medical systems for exchanging patient's medical data, namely: patient reception or registration, referencing to specialized consultants, processing of various types of requests, planning of medical supplies, recording the results of the treatment process, conducting clinical observations, patient monitoring, and so on.

\subsection{DICOM Standard}

Another transmission standard that is widely used by medical equipment and software producers is DICOM (Digital Imaging and Communications in Medicine), was developed by the National Electrical Manufacturers Association and continues to improve [11; 12], see Fig. 2.

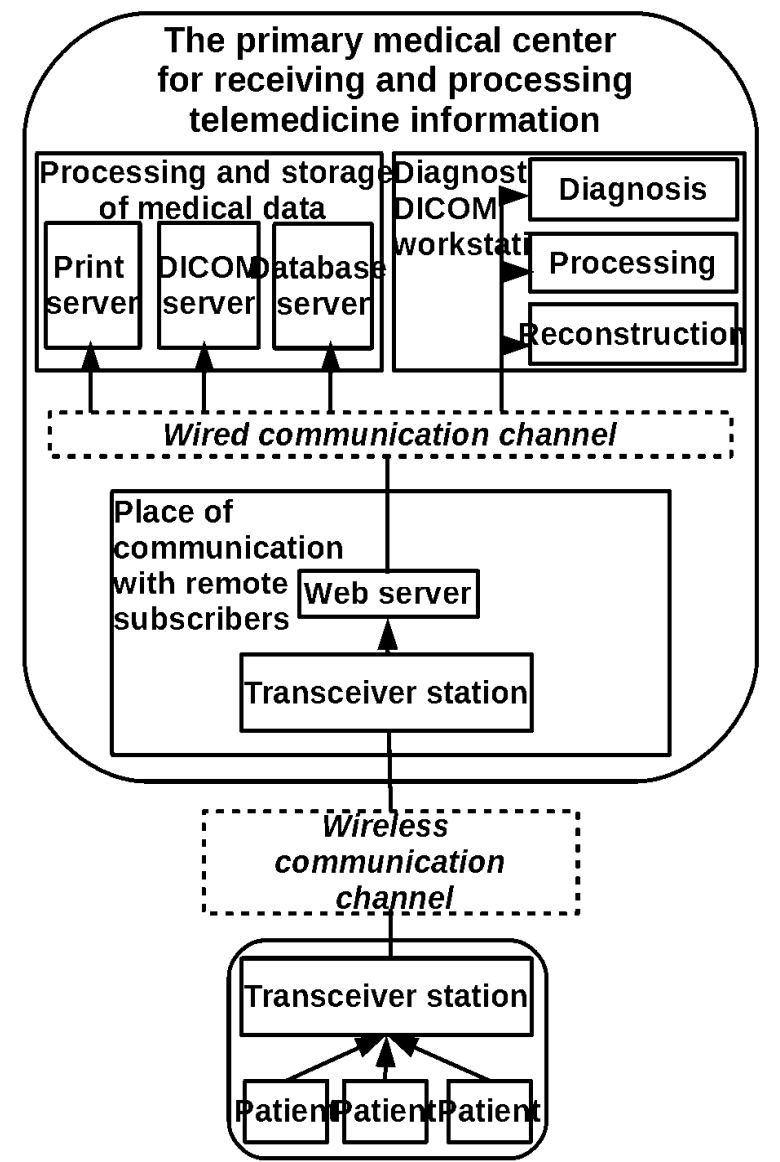

Fig. 2. Structural-Logical Scheme of Implementation of Medical ITS Based on DICOM Protocol.
The DICOM standard allows one to create, store, transmit and print selected images, sequences of images, information about the patient, medical institution, the medical staff that conducts treatment, existing equipment, and so on (see Table 1).

ITSs based on DICOM protocol are client-server structures consisting of interlinked components and software [12]:

- medical DICOM equipment, playing the role of DICOM clients;

- web server to support the client-server structure;

- workstation for medical survey and data processing (DICOM automated diagnostic station, single or a branched system);

- DICOM peripheral equipment.

The DICOM standard provides a digital communication system between DICOM server and diagnostic equipment, including [12]: X-ray equipment (RF, CF, DF, RG, MG, CR); angiographic complexes (XA, DSA); computed tomography (CT, PET); magnetic resonance tomography (MR); isotope scanners (NM); ultrasound scanners (US, EC); microscopes (DM); endoscopes (ES).

Table 1. DICOM Services.

\begin{tabular}{|c|c|l|}
\hline No & $\begin{array}{c}\text { Name of the } \\
\text { DICOM service }\end{array}$ & \multicolumn{1}{|c|}{ Function of the DICOM service } \\
\hline 1 & Store & $\begin{array}{l}\text { Processing and storage of visual and } \\
\text { other telemedicine information }\end{array}$ \\
\hline 2 & File & $\begin{array}{l}\text { Data storage on data carriers for } \\
\text { digital data exchange }\end{array}$ \\
\hline 3 & $\begin{array}{l}\text { Storage Service } \\
\text { Class Provider }\end{array}$ & $\begin{array}{l}\text { Software (client-server architecture) } \\
\text { for organizing DICOM network }\end{array}$ \\
\hline 4 & $\begin{array}{c}\text { Storage Service } \\
\text { Class User }\end{array}$ & $\begin{array}{l}\text { Customer software for interacting } \\
\text { with DICOM network }\end{array}$ \\
\hline 5 & Query/Retrieve & Exchange of patients' history and lists \\
\hline 6 & Modality Worklist & $\begin{array}{l}\text { Creation of lists of conducted and } \\
\text { necessary patients' health tests }\end{array}$ \\
\hline 7 & Print & $\begin{array}{l}\text { Provision of interaction between } \\
\text { software and specialized peripheral } \\
\text { DICOM equipment for printing } \\
\text { telemedicine information }\end{array}$ \\
\hline
\end{tabular}

The DICOM standard enables the integration of medical equipment of different classes, including DICOM scanners, DICOM servers, workstations, and DICOM printers into a single medical information and communication network.

\section{Interaction Models}

Several different classifications of medical ITSs can be distinguished: by the principle of operation or scope of application and by the level of placement in the health care system. There are the following levels [3; 4]: 1 - the level of the country's institutional system (hospitals, clinics, mobile laboratories, and so on), 2 - the essential (clinical) level (doctors of various fields and profiles), 3 the local level of the medical system (profiled and specialized medical services and regional management entities), 4 - the regional level of the country's activities (regional institutions). 
At the essential (clinical) level, medical ITSs can be segmented into two main groups [3; 12]:

- Remote counseling, diagnosis, and staff training - the patient's automated workstation is connected to $N$ consultants' workplaces.

- Remote monitoring and registration of patients' vital functions - the consultant's automated workstation is connected to personal medical devices of $N$ patients.

Despite the diversity of medical ITSs, they are characterized by typical operations related to registration, transmission, processing, and analysis of data. In general terms, according to [13-15], the structure that processes and transmits information in standard medical ITS can be described as follows.

Analog video or audio signals from any information source (diagnostic equipment, scanners) are received on the input of medical ITS. The signal is sampled based on the frequency characteristics of the equipment and the network, then quantized and becomes a subject to noiseresistant encoding and compression, regarding the network data rate requirements. The signal is then fed to the data-transmitting unit that provides modulation of a certain type, which further becomes the subject of optimal filtering on the receiver side.

The modulated information signal, spreading over the data channel, is distorted by an interference of artificial or natural origin. These interferences contain, as fluctuating, focused or pulsating components and distort the message. Taking account of that the information message is distorted during transmission, an important decision for the ITS functioning is to combine optimal filtering and noise-resistant decoding units in the receiver.

\section{Conclusion}

The provision of high quality of ITS functioning is possible using the noise-resistant methods and structures of the network organization. The need to ensure the efficient transmission of large volumes of medical data in noisy conditions with low signal-to-noise ratios requires the improvement of existing and the development of new methods and models for transmitting and receiving data.

Mentioned circumstances cause the efficiency of the functioning of medical ITS and its ability to provide desired medical services in emergencies. The development and implementation of a unified interaction model for various medical ITS components, which will allow adapting to extreme characteristics of data transmission physical environment, is a vital scientific and practical issue.

\section{References}

1. E. Coiera, Guide to Medical Informatics, the Internet and Telemedicine (Chapman and Hall Medical, London, 1997)

2. V. M. Bezruk, I. P. Nikolaev, D. V. Chebotareva, Information and Telecommunication Signal Recognition Technologies in Telemedicine, Bulletin of Lviv Polytech. National Univer.: Inform. Sys. and Net., 805, 17-22 (2014)

3. V. N. Konyukhov, Fundamentals of Telemedicine Systems (Samara State Aerospace University, Samara, 2012)

4. A. V. Vladzimirskiy, Telemedicine in the System of Health Care Management and Organization (Digital PH, Donetsk, 2012)

5. N. A. Lysov, Y. L. Minayev, V. S. Vopilin, A. A. Supilnikov, A. V. Chebykin, Medical Informatics (Reaviz, Samara, 2013)

6. Y. E. Lyah, A. V. Vladzimirskiy, Introduction to Telemedicine. Series: Essays of Biological and Medical Informatics (Lebed, Donetsk, 1999)

7. S. S. Furuie, et al., Managing medical images and clinical information: InCor's experience, IEEE Transactions on Inform. Tech. in Biomed., 11, 1, 17-24 (2007)

8. V. A. Gerasevich, Computer for Doctor (BHVPetersburg, St. Petersburg, 2004)

9. A. G. Faibushevich, V. D. Protsenko, Application of Clinical Informatics Methods in Complex Studies and Treatment of Patients (PFUR, Moscow, 2008)

10. J. Quinn, HL7, http://www.hl7.eu/HL7v2x/v27/ std27/ch01.html, accessed 2019/10/16 (2011)

11. J. Rodrigues, Health Information Systems: Concepts, Methodologies, Tools, and Applications (IGI Global, Hershey, 2010)

12. V. N. Kazakov, V. G. Klimovitskiy, A. V. Vladzimirskiy, Telemedicine (Nord, Donetsk, 2002)

13. G. F. Konakhovich, I. O. Machalin, O. Yu. Puzyrenko, The Theory of Electrical Communication, (Interservice, Kyiv, 2013)

14. O. N. Novoselov, A. F. Fomin, Fundamentals of the Theory and Calculation of Information-Measuring Systems (Mashinostroenie, Moscow, 1991)

15. R. W. Heath Jr., Introduction to Wireless Digital Communication: A Signal Processing Perspective (Prentice Hall, Upper Saddle River, 2017) 